\title{
Indicadores socioambientais de focos do Aedes aegypti no extremo sul de Santa Catarina
}

\author{
Ivan Merêncio ${ }^{a *}$, Fabiane Tascab $^{\mathrm{b}}$, Carlos Antônio Oliveira Vieira ${ }^{\mathrm{a}}$ \\ a Programa de Pós-Graduação em Engenharia de Transportes e Gestão Territorial, Universidade Federal de Santa Catarina, Santa \\ Catarina, Florianópolis, 88040-970, Brasil. *ivan_merencio@hotmail.com \\ b Programa de Pós-Graduação em Engenharia Ambiental, Universidade Federal de Santa Catarina, Santa Catarina, Florianópolis, \\ 88040-970, Brasil.
}

Recebido: 21 fevereiro 2018 / Aceito: 21 maio 2018 / Publicado online: 28 maio 2018

\begin{abstract}
Resumo
Esta pesquisa analisou a distribuição espacial de focos do Aedes aegypti encontrados em 2016 na área de abrangência da Associação de Munícipios do Extremo Sul Catarinense (AMESC) e comparou com indicadores socioambientais (precipitação, temperatura, saneamento básico e população). Todos os dados são secundários, disponibilizados por diferentes órgãos governamentais. Foram observados 44 focos distribuídos em 6 municípios, sendo que o município Passo de Torres concentrou $61 \%$ desses focos. Esta cidade faz fronteira com o Estado do Rio Grande do Sul, região afetada, e, assim como os demais municípios com alto índice de infestação, é atravessada longitudinalmente pela BR-101. Isto pode indicar que a BR-101 é o principal meio de dispersão do vetor. Há maior ocorrência do vetor nas estações mais quentes (verão e outono), entretanto, não foi observada correlação com a precipitação, ainda que em 2016 esta superou a média histórica da região. Apesar da boa cobertura do abastecimento de água, não há dados sobre resíduos, tampouco sobre o esgotamento na região. Assim, a indisponibilidade de dados específicos de saneamento prejudicou uma análise detalhada de aspectos relacionados a esse indicador. Embora estas informações possam ser utilizadas pelos gestores públicos para definição de diretrizes de combate ao mosquito, são necessárias maiores complementações em nível local.
\end{abstract}

Palavras-chave: Meio Ambiente, saneamento básico; saúde pública.

\section{Socio-environmental indicators of Aedes aegypti breeding sites in the southern extremity of Santa Catarina state}

\begin{abstract}
This research analyzed the spatial distribution of Aedes aegypti breeding sites found in 2016 in the fifteen cities of the Municipalities Association of the Extreme South of Santa Catarina (AMESC). These data were compared with socioenvironmental indicators (rainfall, temperature, basic sanitation and population). All data are secondary, available by different government agencies. A total of 44 mosquitoes breeding sites were observed throughout 6 cities, and the Passos de Torres city concentrated $61 \%$ of them. This city has a frontier with Rio Grande do Sul State, an affected region, and it is crossed longitudinally by the BR-101 highway, as well as other cities with a high rate of infestation. This may indicate that BR-101 is the main means of vector dispersion. A higher occurrence vector in the warmer seasons (summer and autumn) was observed, there was no correlation to precipitation, although it rained more in 2016 than the historical average to the study area. Despite the good coverage of the water supply, there is no data about solid waste for the cities most affected, nor about the sanitary sewage in the region. So, the unavailability of specific data on sanitation impaired a detailed analysis about this indicator. This information can be used by public managers for the definition of Aedes aegypti control guidelines, but more complementarities are needed at the local level.
\end{abstract}

Keywords: Environment, basic sanitation, public health.

\section{Introdução}

Dentre os agravos que acometem a saúde da população brasileira, destaca-se a dengue, uma doença infecciosa cujo espectro clínico pode variar de infecções assintomáticas até quadros graves e fatais. A transmissão do vírus da dengue ocorre por intermédio do inseto vetor, os mosquitos hematófagos do gênero Aedes (Meigen, 1818), com destaque para o Aedes aegypti, (Linnaeus, 1762; Segurado et al., 2016). Mosquitos deste gênero, além da dengue, transmitem outros tipos de doenças, como a febre amarela, chikungunya e zika (Heckmann, 2011), as quais também podem ser encontradas no Brasil. 
A dengue é considerada endêmica, principalmente em regiões tropicais e subtropicais, afetando principalmente as pessoas que vivem em áreas urbanas (Heckmann, 2011). É uma das principais doenças infecciosas presentes no Brasil, surgindo sazonalmente, com maior aparecimento de casos nos primeiros cinco meses do ano, período mais quente e úmido (Viana e Ignotti, 2013).

Somente em 2016 foram registrados pouco mais de 1,5 milhão de casos prováveis de dengue no país, ano em que também se observa o crescimento da chikungunya e o aparecimento da zika (BRASIL, 2017a). Já a febre amarela, considerada endêmica na região amazônica, também foi encontrada no Distrito Federal e nos Estados de Goiás, Mato Grosso do Sul, Minas Gerais, Pará, São Paulo e Tocantins no ano de 2016. Entre julho de 2014 e dezembro de 2016 foi confirmado um total de 15 casos humanos de febre amarela (BRASIL, 2017b), sendo que em 2017 esse número já passa de 792, o maior surto das últimas décadas (BRASIL, 2017c).

Fatores climáticos, crescimento populacional desordenado, migração rural-urbana, condições precárias de saneamento básico e inadequação de infraestrutura das cidades são algumas das condições favoráveis ao desenvolvimento do vetor A. aegypti (Costa et al., 2008; Flauzino et al., 2011), e consequentemente da transmissão das doenças. Com tantos fatores propícios ao desenvolvimento do mosquito, o controle de sua população é complexo. Este panorama de complexidade também é observado no Estado de Santa Catarina.

No ano de 2016, o Estado teve a sua pior marca histórica do registro da dengue. Foram identificados 7.006 focos do mosquito e registrados 4.379 casos da doença, dos quais $92 \%$ são autóctones, ou seja, as pessoas foram infectadas dentro do território catarinense (DIVE/SC, 2017). De modo oposto, $85 \%$ (76) e $83 \%$ (49) dos casos confirmados de chikungunya e zika, respectivamente, foram importados de outros Estados. No ano seguinte, em 2017, o número de focos aumentou em $65,1 \%$ (11.567), mas a notificação das doenças foi significativamente menor, apenas 16 casos confirmados (DIVE/SC, 2018).

Nesse sentido, os estudos de revisão relacionados com o controle vetorial do mosquito são de grande importância para o planejamento estratégico, pois permitem direcionar as medidas dos programas de vigilância em saúde (Zara et al., 2016). Nos casos autóctones, o estudo local ganha importante destaque. É nessa escala geográfica que o processo de transmissão da doença ocorre, permitindo a observação de variáveis e indicadores que, em outros níveis de análise, não seriam perceptíveis (Flauzino et al., 2011).

Flauzino et al. (2011) também destacam que a busca da associação da ocorrência da dengue com condições socioeconômicas e ambientais tentam explicar este panorama de complexidade, mas que nem sempre encontra resultados esperados e concordantes.

Nesta perspectiva o presente estudo analisou a distribuição espacial de focos do A. aegypti encontrados no ano de 2016 na área de abrangência da Associação do Extremo Sul Catarinense (AMESC), comparando esses resultados com indicadores socioambientais.

\section{Material e Métodos}

Para análise de características locais que possam contribuir no desenvolvimento de focos do A. aegypti considerou-se como área de estudo uma associação de municípios, a AMESC (Associação dos Municípios do Extremo Sul Catarinense). A região é formada por 15 municípios (Figura 1), os quais totalizam cerca de 199 mil habitantes (IBGE, 2017).
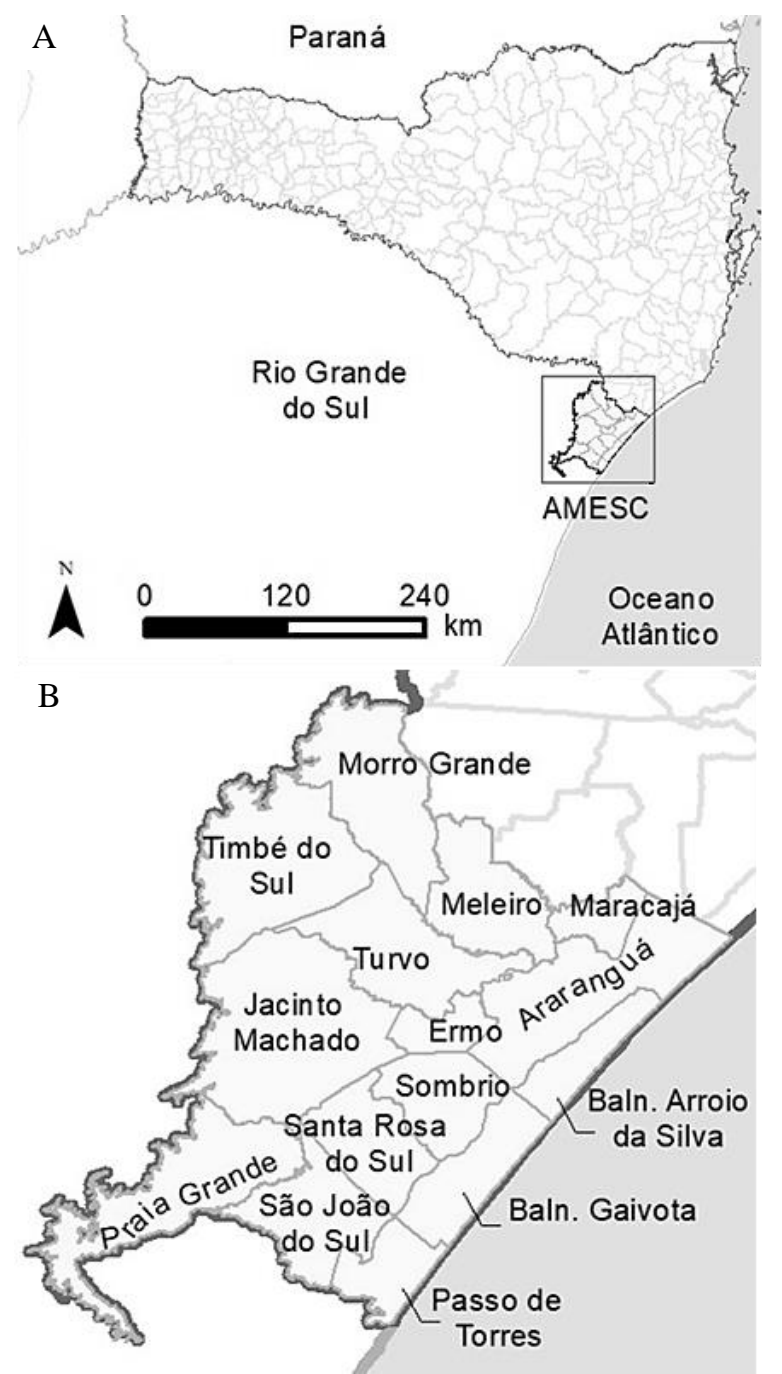

Figura 1. Mapa de Localização da Microrregião da Associação de Munícipios do Extremo Sul Catarinense - AMESC (A) e os municípios que a constitui (B).

Foram pesquisados os registros de focos de mosquitos para o ano de 2016, cujos dados foram cedidos pela DIVE (Diretoria de Vigilância Epidemiológica) de Santa Catarina. Esses dados foram relacionados às condições físicas (precipitação e temperatura) e sociais (saneamento básico e população) relativas ao ano de 2016 nestes municípios (Tabela 1). Utilizou-se dados vetoriais contendo os perímetros municipais e o traçado da Rodovia BR-101.

O tratamento estatístico dos dados da planilha eletrônica foi realizado utilizando o software Microsoft Excel, no qual se utilizou o coeficiente de correlação de Pearson (r) para verificar a correlação entre a precipitação, temperatura e total de focos nas estações do ano de 2016 para a região. 
Tabela 1. Indicadores pesquisados para a região da Associação de Munícipios do Extremo Sul Catarinense (AMESC).

\begin{tabular}{lc}
\hline \multicolumn{1}{c}{ Dados } & Fonte \\
\hline Focos A. aegypti & DIVE (2016) \\
Precipitação média* & Hidroweb (2016) \\
Temperatura média** & INMET (2016) \\
Perímetros Mun. e BR-101 & IBGE (2010) \\
Normais climatológicas & INMET (2017) \\
Estimativa Populacional & IBGE (2016) \\
Saneamento básico & SNIS (2017) \\
\hline
\end{tabular}

* Estações pluviométricas 2849044, 2949001, 2849019, 2950056 e 2950008. ** Estação meteorológica 83897 (Florianópolis). DIVE Diretoria de Vigilância Epidemiológica, INMET - Instituto Nacional de Meteorologia, IBGE - Instituto Brasileiro de Geografia e Estatística, SNIS - Sistema Nacional de Informações Sobre Saneamento.

\section{Resultados e Discussão}

A distribuição espacial de focos do $A$ aegypti nos municípios da AMESC no ano de 2016 está representada na Figura 2. Foram 44 vetores distribuídos em 6 municípios: Passo de Torres, Araranguá, Sombrio, Santa Rosa do Sul, Turvo e Maracajá, em ordem decrescente de infestação. Os 3 primeiros municípios concentram 93\% de todos os focos e somente a cidade de Passo de Torres teve 27 registros (61\%), sendo o município mais afetado.

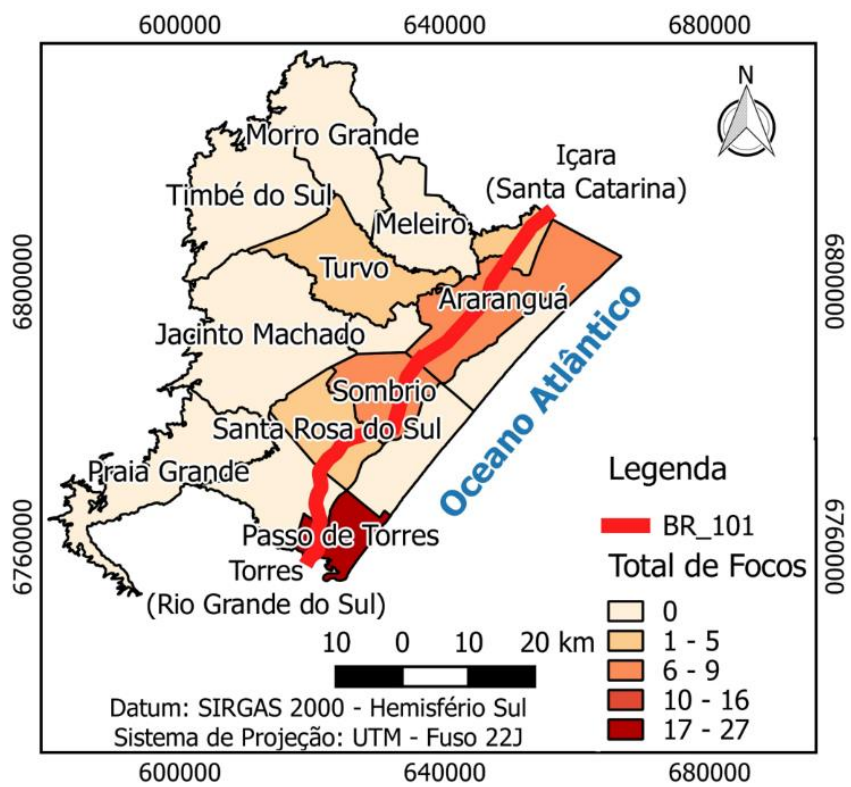

Figura 2: Distribuição espacial do Aedes aegypti na Associação de Munícipios do Extremo Sul Catarinense (AMESC), em 2016.

A dispersão do A aegypti através do voo ocorre em pequenas distâncias, sendo que a fêmea voa em média até 288,12 metros (Freitas e Lorenço, 2009). Nesse sentido, a região nordeste do Rio Grande do Sul foi considerada infestada pelo Aedes aegypti (Figura 3), possuindo 5 casos autóctones confirmados de dengue, incluindo-se nessa estatística o município de Torres, que registrou 1 caso (CEVS, 2017). Observa-se que Torres está situado na fronteira com Santa Catarina (Figura 2), com a cidade de Passo de Torres, sendo em grande parte zonas urbanas. Em 2016, o nordeste do Rio Grande do Sul encontrava-se com um índice de infestação satisfatório, quando menos de $1 \%$ dos imóveis fiscalizados possui larvas do Aedes aegypti, mas com a indicação a presença do vetor nesta região.

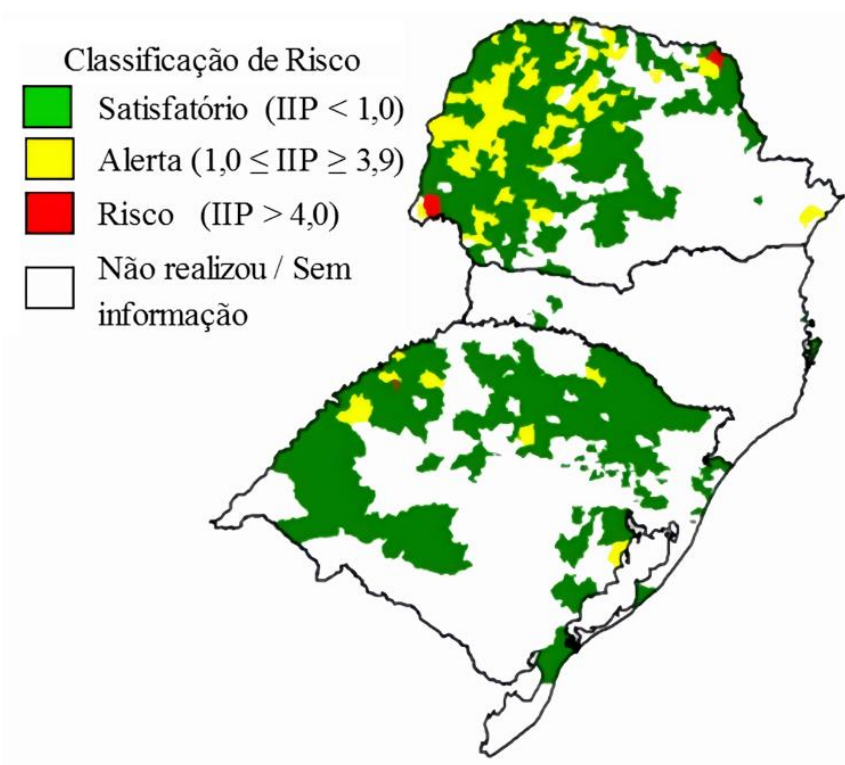

Figura 3: Mapa do índice de infestação da Região Sul do Brasil no Ano de 2016. Fonte: Secretaria de Vigilância em Saúde (2016).

Estudos apontam que o principal meio de dispersão do mosquito para outras regiões é através de meios de transportes que cruzam o país (Schaffner e Mathis, 2014). Neste sentido, observa-se a possível influência da BR-101 na dispersão dos focos através dos municípios infectados, que são atravessados longitudinalmente por esta rodovia (Figura 2), uma via de importância nacional para o transporte de cargas. Dessa maneira, a proximidade com cidades infectadas no Estado do Rio Grande do Sul pode influenciar no surgimento de focos no extremo sul catarinense, transportados pela BR-101.

A média de precipitação, temperatura e total de focos, na AMESC, conforme as estações do ano de 2016, estão descritos na Tabela 2.

Tabela 2. Média de precipitação $(\mathrm{mm})$, temperatura $\left({ }^{\circ} \mathrm{C}\right)$ e total de focos conforme as estações do ano de 2016 na Associação de Munícipios do Extremo Sul Catarinense (AMESC).

\begin{tabular}{lccc}
\hline \multicolumn{1}{c}{ Estação } & $\begin{array}{c}\text { Média de } \\
\text { Precipitação }\end{array}$ & $\begin{array}{c}\text { Média de } \\
\text { Temperatura }\end{array}$ & $\begin{array}{c}\text { Total de } \\
\text { Foco }\end{array}$ \\
\hline Verão & 185,23 & 29,79 & 26 \\
Outono & 80,65 & 23,35 & 16 \\
Inverno & 105,35 & 22,18 & 0 \\
Primavera & 227,57 & 26,39 & 2 \\
\hline
\end{tabular}

O aumento da precipitação é um fator favorável ao crescimento do número de locais contaminados pelo mosquito transmissor da dengue (Leite et al., 2008; Marteis et al., 2013), mas para a área de estudo não foi observada uma correlação direta $(r=0,23)$. A precipitação do ano de 2016 foi comparada 
à média histórica dada pelas normais climatológicas (INMET, 2017), o que demonstrou que o ano de 2016 foi $25 \%$ mais chuvoso do que média anual (Figura 4). Isto reforça a não correlação entre a precipitação e a quantidade de focos encontradas.

Já a temperatura média apresentou uma correlação moderada $(r=0,65)$, o que pode indicar que esta teve influência no índice de infestação. O desenvolvimento do $A$. aegypti é favorecido em temperaturas entre $22^{\circ} \mathrm{C}$ e $36^{\circ} \mathrm{C}$ (Marinho, 2013), e as estações do ano de 2016 tiveram um intervalo ideal para o desenvolvimento do vetor na região. Os focos diminuíram ao longo do ano, principalmente no inverno, estação em que não foram registrados focos, conforme ilustra a Figura 5.

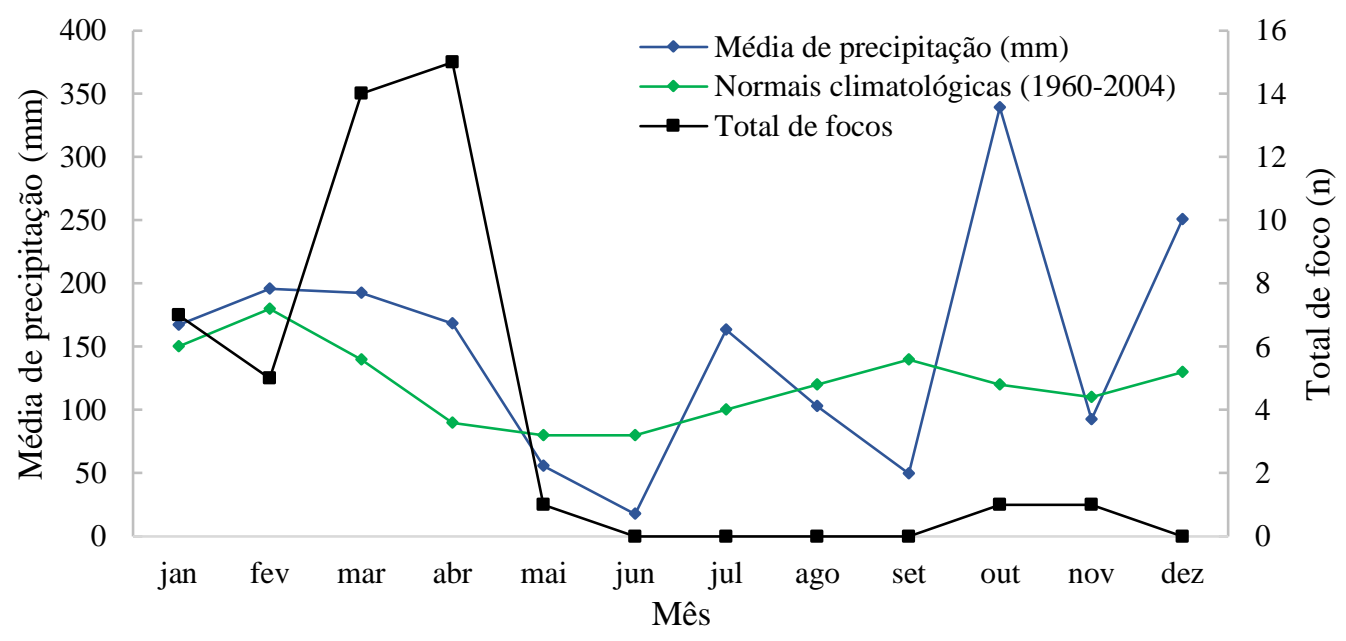

Desta forma, é importante avaliar outros fatores que podem propiciar condições favoráveis ao surgimento do mosquito. Alguns trabalhos evidenciam uma relação positiva entre as condições de vida e a ocorrência da dengue (Scandar et al., 2010; Cabral e Freitas, 2012), tal como a ausência de cobertura de saneamento básico. A oferta irregular do abastecimento de água, a ausência de tratamento de esgoto, a inadequada coleta de lixo e a ausência de estruturas de drenagem urbana favorecem o acúmulo de água parada e podem tornar o ambiente vulnerável à procriação do mosquito (Tabela 3).

Dados do Levantamento Rápido do Índice de Infestação pelo $A$. aegypti (LIRAa) revelam que, na região Sul, 47,3\% dos focos foram encontrados no lixo, enquanto no Nordeste e no Centro Oeste o armazenamento de água é a principal fonte de preocupação com $78,8 \%$ e $36,8 \%$, respectivamente. Já o Norte e o Sudeste têm no depósito domiciliar o principal desafio, com taxas de $46,6 \%$ e $55,1 \%$, respectivamente (BRASIL, 2014). A própria FUNASA (2017) observa que a única forma de 'ganhar a guerra' contra o Aedes é com saneamento.

Analisando os dados do Sistema Nacional de Informações sobre Saneamento (SNIS, 2017) para os municípios da AMESC, observa-se que todas as áreas urbanas são abastecidas com água pela rede pública, mas, apenas uma (Praia Grande) possui dados relativos à coleta de esgoto $(23,31 \%)$. Já a coleta direta de resíduos sólidos na área urbana é existente na maioria dos municípios, mas não há dados disponíveis para as cidades mais afetadas (Passos de Torres e Araranguá), o que não permite relacionar os focos do Aedes e a ausência da coleta de resíduos. Além disso, ainda não há publicado um diagnóstico nacional sobre as águas urbanas, não permitindo um diagnóstico do setor nas cidades da AMESC. Desta forma, observa-se que a indisponibilidade de dados de saneamento é um entrave a um diagnóstico consubstanciado sobre a presença de focos na região.

Tabela 3. Percentual de atendimento urbano de água (\%AA), cobertura de coleta direta de resíduos domésticos relativos à população Urbana (CCD) e total de focos encontrado nos municípios que compõem a AMESC (2016).

\begin{tabular}{lcccc}
\hline \multicolumn{1}{c}{ Município } & $\begin{array}{c}\text { População } \\
2017\end{array}$ & \%AA & CCD & $\begin{array}{c}\text { Total de } \\
\text { Foco }\end{array}$ \\
\hline Araranguá & 67.110 & 100 & - & 8 \\
Sombrio & 29.710 & 98 & 100 & 6 \\
Turvo & 12.746 & 100 & 100 & 1 \\
Baln. Arr. do Silva & 12.344 & 75 & 100 & 0 \\
Jacinto Machado & 10.539 & 80 & - & 0 \\
Balneário Gaivota & 10.413 & 80 & 100 & 0 \\
Passo de Torres & 8.370 & 72 & - & 27 \\
Sta Rosa do Sul & 8.356 & 100 & 100 & 1 \\
Praia Grande & 7.364 & 99 & 92 & 0 \\
São João do Sul & 7.268 & 61 & 100 & 0 \\
Maracajá & 7.139 & 99 & 100 & 1 \\
Meleiro & 7.047 & 99 & - & 0 \\
Timbé do Sul & 5.377 & 100 & 100 & 0 \\
Morro Grande & 2.915 & 92 & 13 & 0 \\
Ermo & 2.076 & 96 & 0 & 0 \\
\hline
\end{tabular}

- Sem indicadores disponíveis.

Observa-se também que não há relação entre o total de habitantes de cada cidade com o total de focos (Tabela 3), já que o município de Passos de Torres possui a maioria dos focos mesmo com apenas a $7^{\mathrm{a}}$ maior população da AMESC. Se esta cidade fosse desconsiderada da análise a correlação entre a população e os focos seria forte, mas a série história aqui apresentada é pequena para inferir neste fato. Há, contudo, algumas suposições que podem ser feitas a partir dos dados 
apresentados.

A fiscalização de focos do mosquito em Passos de Torres pode ser mais efetiva, já que faz fronteira com um município afetado, enquanto a fiscalização em outras cidades pode não ser regular, o que mascara dados reais e poderia justificar a não relação com a precipitação. Sabe-se que em áreas com maior densidade demográfica o mosquito consegue infectar mais gente para um mesmo raio de atuação, haja vista as condições precárias do saneamento que permitem que o ciclo se propague continuamente.

Além disso, apesar de bons índices no que tange o abastecimento de água (90\%) e coleta de resíduos (82\%) nas áreas urbanas, não se pode afirmar que os focos não foram encontrados nas pequenas parcelas sem cobertura. De igual modo pouco pode-se relacionar ao esgotamento sanitário, cuja coleta é praticamente inexistente na região. Essas informações devem ser utilizadas pelos gestores públicos para definição das diretrizes de combate ao mosquito bem como à melhoria dos dados públicos disponíveis, possibilitando propor medidas que aumentem a resiliência da região ao surgimento do $A$. aegypti.

\section{Conclusões}

No presente estudo foram avaliadas algumas características físicas e sociais que podem influenciar no aparecimento de focos de A. aegypti nas cidades do extremo sul de Santa Catarina. Foi observada uma correlação moderada entre os as temperaturas médias mensais e, apesar de ser relatado em estudos diversos a influência da precipitação, este fato não foi evidenciado neste estudo. A ausência de rede coletora de esgoto pode contribuir no surgimento de focos, mas a indisponibilidade de dados sobre coleta de resíduos é um entrave a um diagnóstico consubstanciado ao setor. Foi evidenciado que a malha rodoviária, mais especificamente a rodovia BR 101, pode influenciar no surgimento de vetores, já que foram observados focos nas cidades lindeiras ao eixo rodoviário.

Diversas variáveis relacionam-se com o surgimento de focos do vetor, mas a indisponibilidade de dados, bem como possíveis falhas de registros, podem prejudicar um panorama fidedigno para a região da AMESC. As informações adquiridas podem ser utilizadas pelos gestores públicos para definição de diretrizes de combate ao mosquito, mas são necessárias maiores complementações em nível local.

\section{Referências}

Brasil; 2014. Ministério da Saúde atualiza dados sobre casos de dengue. Acesso em 07/11/2017.

Brasil; 2017a. Ministério da Saúde; 2017a. Boletim Epidemiológico, nº 3, Volume 48. Acesso em 07/10/2017.

Brasil; 2017b. Orientação para profissionais de saúde sobre febre amarela silvestre. Portal da Saúde. Acesso em 12/11/2017.

Brasil; 2017c. Centro de operações de emergências em saúde pública sobre febre amarela. Ministério da Saúde. Acesso em 12/11/2017.

Cabral, J. de A.; Freitas, M.V. de. 2012. Distribuição Espacial e Determinantes Socioeconômicos e Demográficos da Dengue nos Municípios Brasileiros. Revista Brasileira de Estudos Regionais e Urbanos, 6(1): 81-95.

CEVS (Centro Estadual de Vigilância em Saúde); 2017. Informativo Epidemiológico Dengue, Chikungunya, Zika Vírus e Microcefalia. Acesso em 01/10/2017.
Costa, F.S., Silva, J.J.D., Souza, C.M.D., \& Mendes, J. 2008. Dinâmica populacional de Aedes aegypti (L) em área urbana de alta incidência de dengue. Revista da Sociedade Brasileira de Medicina Tropical, 41(3) 30912.

Dive/SC - Diretoria de Vigilância Epidemiológica de Santa Catarina; 2017. Boletim Epidemiológico $n^{\circ}$ 37/2016 Situação da dengue, febre do chikungunya e zika vírus em Santa Catarina. Acesso em 07/11/2017.

Dive/SC - Diretoria de Vigilância Epidemiológica de Santa Catarina; 2018. Boletim Epidemiológico n 26/2017 Vigilância entomológica do Aedes aegypti e situação epidemiológica da dengue, febre de chikungunya e zika vírus em Santa Catarina. Acesso em 14/03/2018.

Flauzino, R.F., Souza-Santos, R., \& de Oliveira, R.M. 2011. Indicadores socioambientais para vigilância da dengue em nível local. Revista Saúde e Sociedade, 20(1) 225-240.

Freitas, R.M. de; Lorenço, R.L. de. 2009. Presumed unconstrained dispersal of Aedes aegypti in the city of Rio de Janeiro, Brazil. Revista Saúde Pública, 43(1) 8-12.

FUNASA - Fundação Nacional de Saúde; 2017. Portal do Saneamento Básico. Acesso em 01/08/2017.

Gianuca, K.S.; Tagliani, C.R.A. 2011. Análise em um Sistema de Informação Geográfica (SIG) das alterações na paisagem em ambientes adjacentes a plantios de pinus no Distrito do Estreito, município de São José do Norte, Brasil. Revista de Gestão Costeira Integrada, 12(1) 43-55.

Heckmann, M.I.O. 2012. Dengue: Aspectos Epidemiológicos e o primeiro surto ocorrido na região do Médio Solimões, Coari-Amazonas, no período de 2008-2009. Revista da Sociedade Brasileira de Medicina Tropical, 44(4) 471-474.

Hidroweb; 2016. Séries Históricas. Acesso em 02/08/2017.

IBGE Cidades; 2017. Estimativa populacional de 2017. Acesso em 02/07/2017

Inmet; 2017. Normais climatológicas para o período 1960 - 2004. Acesso em 23/Mai/2017.

Leite, M. E., Fonseca, D. D. S. R., \& Braz, C. K. R 2008. Uso do SIG na análise da dengue: aplicação na microrregião de Montes Claros/Bocaiúva, Minas Gerais, Brasil. Hygeia: Revista Brasileira de Geografia Médica e da Saúde, 3(6) 126-141.

Marinho, R.A. 2013. Ecobiologia de Aedes aegypti (L.1762) (Diptera: Culicidae) associada a fatores climáticos em três mesorregiões da Paraíba. Dissertação de Mestrado, Programa de Pós Graduação em Ecologia e Conservação / Universidade Estadual da Paraíba , Campina Grande, Paraíba. 76p.

Marteis, L.S., Steffler, L.M., Araújo, K.C.G.M.D., \& Santos, R.L.C.D. 2013 Identificação e distribuição espacial de imóveis-chave de Aedes aegypti no bairro Porto Dantas, Aracaju, Sergipe, Brasil entre 2007 e 2008. Cad. Saúde Pública, 29(2) 368-378.

Scandar, S.A.S., Vieira, P., Cardoso Junior, R.P., Silva, R.A.D., Papa, M., \& Sallum, M.A.M. 2010. Dengue em São José do Rio Preto, Estado de São Paulo, Brasil, 2005: fatores entomológicos, ambientais e socioeconômicos. BEPA, Bol. epidemiol. paul. 7(81) 04-16.

Schaffner, F.; Mathis, A. 2014. Dengue and dengue vectors in the WHO European region: past, present, and scenarios for the future. The Lancet Infectious Diseases, 14(12) 1271-1280.

Segurado, A.C., Cassenote, A.J., \& Luna, E.D.A. 2016. Saúde nas metrópoles - Doenças infecciosas. Estudos Avançados. Revista Estudos Avançados, 30(86) 29-49.

SNIS - Sistema Nacional de Informações Sobre Saneamento; 2017. Acesso em 07/Nov/2017

Viana, D.V.; Ignotti, E. 2013. A ocorrência da dengue e variações meteorológicas no Brasil: revisão sistemática. Revista Brasileira de Epidemiologia, 16(2) 240-256.

Zara, A.L.D.S.A., Santos, S.M.D., Fernandes-Oliveira, E.S., Carvalho, R.G., \& Coelho, G.E. 2016. Estratégias de controle do Aedes aegypti: uma revisão. Epidemiologia e Serviços de Saúde, 25(2), 391-404. 